\title{
The working zone in the interpolar area of the Faraday balance: an approach to testing the magnetic force factor stability criterion
}

\author{
Alexander V. Sandulyak, Anna A. Sandulyak, Maria N. Polismakova, Dmitry O. Kiselev, Darya A. Sandulyak, Vera A. \\ Ershova
}

Moscow Technological University, 107996 Moscow, Stromynka, 20, RF

\begin{abstract}
Due to rapid development of the scientific and applied research in magnetic control and magnetophoresis of ferro- and ferromagnetic disperse fraction of various technological, natural and industry-specific media, the Faraday method is again in high demand as it is mainly aimed at defining magnetic susceptibility of solid and heterogeneous samples of small volumes. Based on the appearing (and then measured) ponderomotive force impacting the sample, the method allows accurate determining of single particles magnetic susceptibility by using the data of the sample represented by a conglomerate of the particles of such a fraction. In addition, it is mentioned that to date there is still a great gap in the methodology of the Faraday method as there are no exact recommendations on choosing both the form of the polar pieces of the Faraday balance and the positioning of the sample (the location of the working zone) in the interpolar area. Owing to these drawbacks, the well-known and long-time used Faraday method cannot be considered substantiated to a satisfactory degree. Thus, in our point of view, the treatment of the results obtained earlier with the help of the method should be cautious. In our work, we experimentally defined and substantiated an approach to identifying a working (local) zone, viz. the zone with stable values of the magnetic force factor - the product of the field intensity and induction by its gradient. The approach features the relative phenomenological analysis and is exemplified by polar pieces of non-traditional spherical form. It has been demonstrated that in order to state the fact of mere existence of this zone (and its location) in the interpolar area, the coordinate (usually nonlinear one) characteristic of intensity or induction, which is obligatorily obtained in an experiment, should have an inflexion, which guarantees a functionally extreme view of the following coordinate characteristics both of the gradient and that of the force factor. We also established the coordinates and the length of the working zone in a specific interpolar area.
\end{abstract}

\section{Introduction}

Faraday ponderomotive method is one of the classical techniques used to define magnetic susceptibility $\chi$ of solid and heterogeneous samples of low volume $V$. The method is based on measuring the magnetic (ponderomotive) force $F$ affecting the studied sample that is placed in an inhomogeneous field between the polar pieces of the Faraday balance; the method presupposes employing the well-known dependence $F=$ $\mu_{0} \cdot \chi \cdot V \cdot \operatorname{HgradH}=\chi \cdot V \cdot \operatorname{BgradB} / \mu_{0}\left(\right.$ where $\mu_{0}=4 \pi \cdot 10-7 \mathrm{H} / \mathrm{m}$ is a magnetic constant) to define values $\chi$.

Despite available alternative yet not always liveable solutions, this method can be given a new perspective of its targeted practical application. We are talking about the advancing applied scientific research in magnetophoresis and magnetic control of highly dispersed ferro- or ferrimagnetic particles (ferrofractions of many technological, natural and industry-specific media) [1-6], where one of the key issues is to obtain the data on the particles magnetic susceptibility.
So, by the yielded (almost direct) results of the susceptibility of the sample represented by a conglomerate of the particles experimentally separated from some medium and necessary to realise the Faraday method, we can quite definitely estimate the susceptibility of single particles by dividing these data by the values of their packaging bulk density (under condition of their sufficient mutual separation).

However, despite the Faraday method having been used by many generations of researchers, it still remains underdeveloped and thus not accurate enough. Thus, in our opinion, the attitude and treatment of the data array on the Faraday method and its outcomes accumulated and elucidated in the special literature should be cautious.

The point is when implementing the Faraday method, as a rule, one of the key requirements is not observed, the one that calls for rigorous positioning of the studied sample in the zone of the Faraday balance interpolar area. Unlike somewhat simplified opinion according to which this zone should just feature a constant value of the intensity $H$ gradient, i.e. $\operatorname{grad} H$ (or the value of induction $B$ gradient $B=\mu_{0} H$, i.e. grad $B$ ), a more fastidious attitude 
to the working zone would assume preserving the constant value of the united parameter - a product of $H \cdot \operatorname{grad} H$, or $B \cdot \operatorname{grad} B$. However, there is unfortunately very little consideration given either to the issue of substantiated choice of the polar pieces shape (that are capable of ensuring the existence of the working zone), or to the matter of experimental and/or theoretical defining of the location of this zone, e.g. a zone with a stable value of the force factor. The features prerequisite for defining these conditions are virtually absent; the available recommendations are quite vague and hardly suitable for specific practical application.

\section{Conceptual approach}

To give an unbiased estimation on the acceptability of the polar pieces choice for the Faraday balance (their capacity to create a working zone), we must have at our disposal an expanded coordinate of the force factor characteristic (in the chosen direction $x$ permeating the interpolar area e.g. in the line of ponderomotive force). In this case, if we detect any stable (constant or close to constant) values of HgradH or BgradB, we can clearly state the presence of the working zone of our interest in the interpolar area. Hence, it would simultaneously prove the acceptability of the chosen polar pieces for the Faraday balance, if otherwise, the polar heads should be deemed inadequate for this application. The coordinates of location of the detected segment would be the ones which are to be observed when positioning the sample to perform the measurements by the Faraday balance.

Obtaining the needed coordinate characteristic of the force factor HgradH or BgradB is possible after getting the corresponding coordinate characteristic of parameter $H$ or $B$. Indeed, such a characteristic, specifically, parameter $B$, defined e.g. by small stepwise movement of the Hall sensor in the chosen direction $x$ and represented in graphic and/or analytical form, allows us to find the coordinate characteristic of the gradient, i.e. parameter $\operatorname{grad} B=d B / d x$, and then the analyzed parameter $B \cdot \operatorname{grad} B$.

Alongside with that, basing on that requirement for the working zone as the one of stable values of the force factor (at the definite, even comparatively short segment of the coordinate characteristic of that factor), we cannot but acknowledge the following condition, which greatly hampers satisfying this requirement. So, for polar pieces of traditional and specific shapes, the coordinate characteristics of $B$ or $H$ in the expanded view, i.e. the ones obtained when $x$ varies in the range of the interpolar area as a whole, are objectively nonlinear as a rule. This means that expanded characteristics of $\operatorname{gradB}$ and BgradB are highly unlikely to be linear and all the more so self-similar or close to self-similar ones.

This observation can create an impression that it is problematic or even fundamentally impossible to obtain such a coordinate characteristic BgradB at which we would explicitly see the desired limited segment (commensurable to the sample dimensions) featuring comparatively stable values of BgradB or HgradH.
Meanwhile, it is possible to provide a rational solution to this seemingly complex problem already at the stage of initial diagnostics of the tested polar pieces, i.e. at the stage of obtaining preliminary key coordinate characteristics $B$ or $H$. Thus, judging by the layout of any of the yielded characteristics, it is easy to take a decision on, first and foremost, the first issue mentioned, that is the choice (and principal applicability) of the polar pieces. It makes sense to move on to the next question only if we get positive results on this one. Only then, we can start defining characteristics $\operatorname{gradB}, \operatorname{BgradB}$, and finally, employing the required characteristic $B g r a d B$ we can determine the positioning of the working zone.

Unconditionally positive this solution can be only in the case when the obtained characteristic $B$ or $H$ is sinuous, i.e. it has an inflexion. Then its segment (short relatively to the entire expanded nonlinear characteristic) in the vicinity of the inflexion point can be considered close to a linear one, and the values $\operatorname{gradB}(\operatorname{gradH})$ will be practically stable. In this case, it is highly probable to expect that the force factor characteristic, i.e. the product $B \cdot \operatorname{grad} B$, will have an extremum (the abscissa of which is surely different from the abscissa of the $\operatorname{gradB}$ characteristic extremum), in the proximity of which the values BgradB $(\mathrm{Hgrad} H)$ are virtually stable as well, as has been said earlier.

The example is nonlinear characteristics of the field intensity $H$ with a pronounced inflexion in the gap areas between opposing spherical surfaces in the direction $x$, normal towards the centre-to-centre line of balls chain (with its magnetization by the field with intensity $H_{0}<<$ $H$ ). At the characteristic $\operatorname{gradH}=d H / d x$ in the point corresponding to the abscissa of the characteristic $H$ inflexion, there is observed a pronounced extremum; moreover, characteristic HgradH really becomes also extreme (let us note that the extremum abscissa is shifted to the area of lesser values of $x$ ) [7-9].

From the perspectives of a guaranteed creation of a working zone fulfilling the requirement for preserving the values of the force factor HgradH (or BgradB) stable, the considered illustrative example evidently bespeaks the full applicability of the spherical polar pieces use for the Faraday balance (clearly, as mutually disconnected semispherical bodies, shown on Fig.1).

\section{Research results and their discussion}

The practicability of choosing spherical polar pieces for the Faraday balance is proven by the results of special experiments conducted when using $100 \mathrm{~mm}$ semispherical polar pieces (Fig.1) mutually distanced at $10 \mathrm{~mm}$ (along the axial line of the poles) in the magnetic loop of the Faraday balance, with the magnetising force of the electromagnet equalling 12000A.

Fig. $2 a$ (dots) shows the data on measuring induction $B$ obtained with the help of the Hall sensor at different distance $x$ from the axial line. The inflexion of the obtained coordinate characteristic $B$ (by $x$ ) is clearly observed. It means, according to the afore-stated conceptual approach, that in the vicinity of the inflexion 


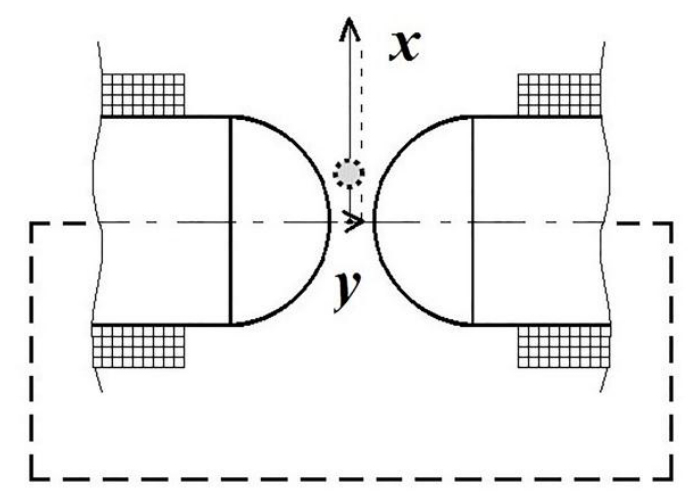

Figure 1. An illustration of one of the polar tips for Faraday balance. The spherical shape allows to create local (settling down in the neighborhood of an extremum of a power factor) a working zone.

point of dependence $B$ on $x$, it is possible to linearly approximate the $B$ data, as is shown in Fig. $2 a$ by dashed lines (here for the illustration purposes they are a little bit longer than the segment of the linear approximation). It at the same time testifies the extreme view of characteristics $\operatorname{grad} B$ and quite probably, can be indicative of the extreme view of characteristics $\operatorname{BgradB}=d B / d x$.

To prove this and to obtain the necessary sufficient case-based quantitative evidence, we first performed analytical (phenomenological) approximation of the nonlinear characteristic $B$ obtained experimentally (Fig. $2 a$, dots) by the $4^{\text {th }}$ degree polynom:

$$
B=a_{1} x+a_{2} x^{2}+a_{3} x^{3}+a_{4} x^{4}+a_{0} .
$$

With the established (with the help of appropriate software, e.g. Excel, Advanced Grapher, MathCad etc.) individual values of coefficients $a_{1}, a_{2} a_{3}, a_{4}$, and $a_{0}$, the initial experimental data (Fig. $2 a$, dots) can be described quite efficiently (Fig. $2 a$, line).

Then, using expression (1) and differentiating it, we can easily get an expression for describing coordinate characteristic $\operatorname{grad} B=d B / d x$ :

$$
\operatorname{grad} B=a_{1}+2 a_{2} x+3 a_{3} x^{2}+4 a_{4} x^{3} .
$$

This characteristic gradB illustrated in Fig. $2 b$, being quite objective for a big range of $x$, is really extreme (except for the initial and final segments which are of no interest and the unusual flow of which is stipulated by the peculiarities of the chosen initial polynom, however, they do not influence the result of the formulated problem).

The revealed fact of gradB characteristic extreme view allows us to expect the presence of a similar phenomenon in a more interesting for us characteristic $\operatorname{BgradB}$, for which we should use the following expression:

$$
\begin{gathered}
B \cdot \operatorname{grad} B=B \cdot d B / d x=\left(a_{1} x+a_{2} x^{2}+a_{3} x^{3}+a_{4} x^{4}+a_{0}\right) \mathrm{x} \\
\mathrm{x} \cdot\left(a_{1}+2 a_{2} x+3 a_{3} x^{2}+4 a_{4} x^{3}\right),
\end{gathered}
$$

which is the product of expressions (1) and (2).

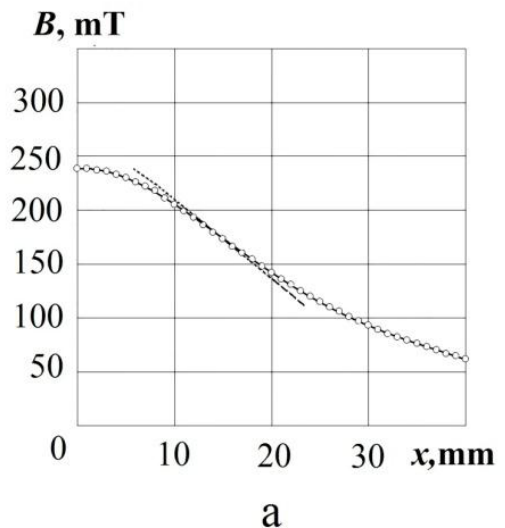

$\operatorname{gradB}, \mathrm{mT} / \mathrm{mm}$
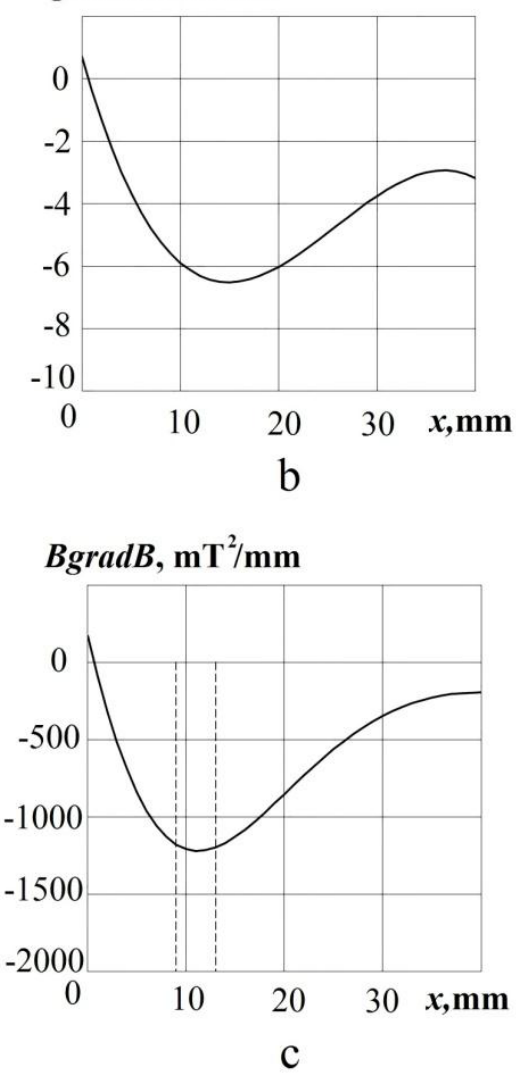

Figure 2. Coordinate characteristics of the field induction (a), its gradient (b), and the force factor (c) between the polar pieces, dots are the experimental data, lines are the calculations.

Fig. $2 c$ illustrates the coordinate characteristic BgradB obtained with the help of (3). As has been expected, this characteristic also has an extreme view. Let us note at that, the value of the extremum abscissa (Fig. 2c) of characteristic BgradB, which is $11.2 \mathrm{~mm}$, is 1.33 times less than the values of the extremum abscissas $\operatorname{grad} B$, which is $14.9 \mathrm{~mm}$

Therefore, the zone near the extremum of coordinate characteristic BgradB, as the area of relatively stable values $\operatorname{BgradB}$, can be considered a working one, i.e. responsible for positioning of the sample studied. At that 
in the area examined (between the given spherical polar pieces) the size of the working zone per se, i.e. the zone of virtually stable values of parameter $\operatorname{BgradB}$ varies (by $x$ ) from $9 \mathrm{~mm}$ to $13 \mathrm{~mm}$. At that, the proper size of the working zone, and consequently, the sample placed in it, should not exceed $4-4.5 \mathrm{~mm}$. It can be clearly seen in Fig. $2 c$, where the corresponding segment $x$ is specially highlighted.

The established sizes of the working area testify only to its "longitudinal" size, namely along the axis $x$ lying in the plane of symmetry of interpolar area (Fig.1). However such information isn't rather complete, it is necessary to have the relevant data on the found zone in its "cross", perpendicular an axis $x$ direction (Fig.1). Receipt of such data requires accomplishment of the researches described above, but in the direction parallel "central" (the symmetry of interpolar area lying in the plane) to an axis $x$; on Fig. 1 it is illustrated by the shaped line remote from the "central" axis $x$ at $y$ distance.

The measurements of induction $B$ taken in the planes parallel to the plane of symmetry of interpolar area and remote from it at various distances of $y \leqslant 3 \mathrm{~mm}$ showed the following. The received characteristics of $B$ were very close to the found characteristic shown on Fig. $2 a$. The excess of each of them testified to a possibility of linear approximation of data in the neighborhood of an excess, showed an extremum of characteristics of $\operatorname{gradB}$ received by the polynomial described characteristics $B$ (1) and their differentiation. Such excess show also the availability of extremum of characteristics of BgradB. At the same time, the proximity of values of abscissa and ordinate of extremum of characteristics $\operatorname{BgradB}$ was noted up to $y=2-2,2 \mathrm{~mm}$. Therefore, the working area in the "cross" direction can constitute at least the size $2 y=$ $4-4,4 \mathrm{~mm}$, i.e. practically as well as in the "longitudinal" direction.

\section{Conclusion}

The paper substantiates and exemplifies by the appropriate choice of perspective polar spherical pieces the approach that has not been analysed yet. This approach allows identifying and diagnosing a local working zone with rigorous spatial constraints aimed at positioning the small-sized samples and studying their magnetic susceptibility in the interpolar area of the Faraday balance, the zone having a stable magnetic force factor at that. It comes down to choosing such a form of polar pieces (in particular, a spherical one) for the interpolar area, that would ensure the received coordinate characteristics of induction (intensity), which are usually nonlinear, are in this case sinuous, i.e. have an inflexion. Then for the zone close to the inflexion point and yielding to linearization, the corresponding derivative would feature an extremum, which in the final result ensures the extreme view of the force factor (and obtaining a working zone in the vicinity of the extremum). It has been established that in the area between spherical polar pieces with diameter of $100 \mathrm{~mm}$ and mutually distanced at $10 \mathrm{~mm}$, the location of the working zone itself, i.e. the zone of practically stable values of parameter BgradB, varies (by $x$ ) from $9 \mathrm{~mm}$ to $13 \mathrm{~mm}$. At that, the proper size of the working zone, and consequently, the sample placed in it, should not exceed $4-4.5 \mathrm{~mm}$.

\section{Acknowledgement}

The research is conducted with financial support from RFFI within the frameworks of research projects № 1638-60034 mol_a_dk, № 16-58-10049 KO_a and from Russian Federation Ministry of Education and Science №9.9626.2017.

\section{References}

1. Patent 4492921 US. Method of Determining the Quantity of Solid Fraction of Ferromagnetic Matter in a Fluid. Sandulyak A.V., Garaschenko V.I., Korkhov O.J. Published 1985.

2. K.Nandy, S.Chaudhuri, R.Ganguly, I.K.Puri. Analytical model for the magnetophoretic capture of magnetic microspheres in microfluidic devices, Journal of Magnetism and Magnetic Materials, 320 (2008).

3. V.Murariu, J.Svoboda, The applicability of Davis tube tests to ore separation by drum magnetic separators, Physical Separation in Science and Engineering, 12 (2003).

4. S.Das, S.Chakraborty, Sushanta. K. Mitra. Magnetohydrodynamics in narrow fluidic channels in presence of spatially non-uniform magnetic fields: framework for combined magnetohydrodynamic and magnetophoretic particle transport, Microfluidics and Nanofluidics, 13, 5 (2012).

5. M.Kawano, H.Watarai. Two-dimensional flow magnetophoresis of microparticles, Analytical and Bioanalytical Chemistry, 403, 9 (2012).

6. A.A.Sandulyak, A.V.Sandulyak, B.M.Fethi Belgacem, D.O.Kiselev. Special solutions for magnetic separation problems using force and energy conditions for ferro-particles capture, Journal of Magnetism and Magnetic Materials, 401 (2016).

7. A. A. Sandulyak, V. A. Ershova. D.V.Ershov, A.V.Sandulyak. On the properties of "short" granular magnets with disordered chains of grains: A field between grains, Physics of the Solid State. 52, 10 (2010).

8. A. V. Sandulyak, A. A. Sandulyak, V. A. Ershova. On the model of channel-by-channel magnetization of a granular medium (with a radial permeability profile of a quasi-continuous channel), Technical Physics, $\mathbf{5 4 , 5}$ (2009).

9. A. V. Sandulyak, A. A. Sandulyak, V. A. Ershova. Magnetization curve of a granulated medium in terms of the channel-by-channel magnetization model (new approach), Doklady Physics. 52, 4 (2007) 